\title{
In-feed Use Route of Administration
}

National Cancer Institute

\section{Source}

National Cancer Institute. In-feed Use Route of Administration. NCI Thesaurus. Code C149576.

Administration of a veterinary medicinal product by incorporation into the animal feed. 